\title{
Calculation of Effective Permittivity and Optimization of Absorption Property of Honeycomb Cores with Absorbing Coatings
}

\author{
Kepeng QIU *, Shuqi FENG, Chen WU, Weihong ZHANG, Zijun LIU \\ School of Mechanical Engineering, Northwestern Polytechnical University, Xi'an, Shannxi, 710072, P.R. China \\ cross $^{\text {ref }}$ http://dx.doi.org/10.5755/j01.ms.22.3.8456
}

Received 13 October 2014; accepted 17 May 2015

\begin{abstract}
This paper focuses on the honeycomb cores with absorbing coatings, the effective permittivity is predicted and the absorption property is optimized. The correctness of effective results is verified by experimental measurement, numerical simulation and theoretical analysis. When honeycomb cores with absorbing coatings are regarded as layered multi-phase materials, the upper and lower bounds of effective permittivity are calculated by the way of multi-step equivalence. In order to exhibit the dispersion effect, the improved strong fluctuation theory is proposed. In the second part of works, the size optimization is executed to improve absorption property. The optimization problem is defined that the area $\mathrm{T}$ which is surrounded by the effective reflectivity curve and the boundary line $\left(R_{0}=-10 \mathrm{~dB}\right)$ is maximized under the upper bound constraints of reflectivity and volume fraction. The optimal effective reflectivity curve is obtained within the given frequency range.

Keywords: honeycomb cores, absorbing coatings, effective permittivity, absorption property.
\end{abstract}

\section{INTRODUCTION}

In recent years, there has been an increase in the demand for electromagnetic absorbing structures that must have high stiffness-to-weight ratios and light weight. The honeycomb cores with absorbing materials are able to meet the requirements of the electromagnetic and mechanical properties, which have been extensively used in different structural applications including low-weight antenna reflectors, radomes and other low observable structural components. They are attracting more and more attentions and researches $[1-3]$. The field responses can be obtained by the full numerical means. However, it is very timeconsuming although this approach is accurate [4]. To simplify calculations on large components, the electromagnetic property of honeycomb cores may be represented by an effective permittivity tensor when the periodic cell sizes of honeycomb cores are small enough in comparison with the wavelength within the given frequency range. Smith et al. [5] calculated the effective electromagnetic parameters of dielectric honeycombs by using the finite-difference time-domain technique with periodic boundary conditions. And they pointed out the impact of electrically large honeycomb cells on effective permittivity failure. The permittivity of a typical twodimensional honeycomb core is invariant along the axis of unit cells [6]. If the periodic cell sizes satisfy the wavelength requirement, the transverse parameters are not the simple spatial averages, but instead the weighted averages with respect to the microstructure field distributions among cell walls, air and absorbing regions [7].

Early in 1972, Spiller [8] has studied the absorbing materials, which were used as the low-loss coatings. The matrix method was employed to calculate the

\footnotetext{
${ }^{*}$ Corresponding author. Tel.: +86-29-88493914; fax: +86-29-88495774

E-mail address: qiukp@nwpu.edu.cn (K.P. Qiu)
}

electromagnetic characteristics of multilayer coatings. Aiming at the single layer RAC (Radar Absorbing Coating), Cao et al. [9] proposed two design rules to gain the suitable electromagnetic parameters according to practically applied background of coatings. Liu et al. [10] adopted the transmission/reflection technique to measure the electromagnetic parameters of single-layer absorbing coatings within the desired frequency range. When the absorbing materials are coated on honeycomb cores, the advantageous electromagnetic and mechanical properties can be also obtained simultaneously. He et al. [11] sprayed the metal magnetic micro-powder (MMP) on honeycomb cell walls. Zhou et al. [12] impregnated the honeycomb frame with absorbing solutions. In the both works, experimental results showed that the electromagnetic parameters of honeycomb cores are determined by the concentration of absorbing materials, coating thickness and honeycomb sizes. Because of the periodicity characteristics of honeycomb cores with absorbing coatings, the homogenization method [4,13-15] based on two-scale asymptotic expansion was used to compute the averaging fields from full-wave electromagnetic simulation and analytical calculation. The computational efficiency is rather low. In the present work, several simple and efficient methods are employed. The strong permittivity fluctuation theory (SPFT) [16-19] with long-wavelength approximation, which was originally developed for the wave propagation in continuous random media, was used to predict the effective permittivity of honeycomb cores with absorbing coatings. Based on the perturbation technique, the improved SFT was proposed to exhibit the dispersion effect. Combined with experimental measurement and numerical simulation respectively, NRW method [20, 21] and scattering parameter retrieval method (SPRM) [22] were adopted to calculate the effective permittivity. Lastly the absorption property of parametric honeycomb cores with absorbing coatings is optimized by using the global optimization method. 


\section{MODEL AND EFFECTIVE CALCULATIONS}

\subsection{Periodic honeycomb cores with absorbing coatings}

As shown in Fig. $1 \mathrm{a}$, the periodic honeycomb cores with absorbing coatings are the standard regular hexagonal structure. The unit cell can be considered as a three-phase medium which is made up of matrix, absorbing coating and air. The cross-section is shown in Fig. $1 \mathrm{~b}$.

The electromagnetic properties of perfectly regular hexagonal cells are transversely isotropic. Along the axis of unit cell, the permittivity is invariant. The unit cell geometry governs the electromagnetic properties of honeycomb cores. In the effective model, the geometrical sizes of unit cell satisfy the wavelength requirement. The side length is taken as $l=5.774 \mathrm{~mm}(l<\lambda / 4, f=12 \mathrm{GHz})$ and the thickness of matrix is taken as $t=1.0 \mathrm{~mm}$ according to the failure example of effective permittivity [5]. The thickness of absorbing coatings is taken respectively as $d=0.225 \mathrm{~mm}, 0.45 \mathrm{~mm}, 0.675 \mathrm{~mm}, 0.9 \mathrm{~mm}, 1.125 \mathrm{~mm}$, $1.35 \mathrm{~mm}, 1.575 \mathrm{~mm}$ and $1.8 \mathrm{~mm}$.

The permittivities of matrix, absorbing coating and air are taken as $\varepsilon_{m}=4.0, \varepsilon_{c}=12-\mathrm{j} 1.0$ and $\varepsilon_{a}=1.0$ respectively. The simulation model is shown in Fig. $1 \mathrm{c}$. The incident wave is along the axis of unit cell.

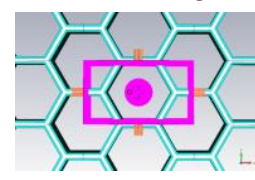

a

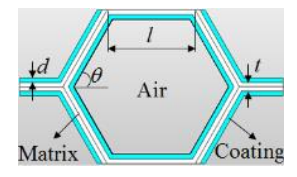

b

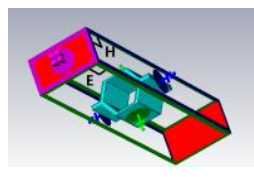

Fig. 1. Periodic honeycomb cores with absorbing coatings

In the following sub-sections, several approximate methods are used to predict effective permittivity. The most widely known analytical approximation was derived by Hashin and Shtrikman [23] using the variational theorems. The strong fluctuation theory (SFT) applied specially to random media [24] is based on the average static field. Both NRW method and numerical simulation are established on the inversion of scattering parameters.

\subsection{H-S bounds}

The Hashin-Shtrikman (H-S) upper and lower bounds are the best obtainable bounds only using material parameters $\varepsilon_{1}$ and $\varepsilon_{2}$ and volume factors $v_{1}$ and $v_{2}$ of twophase medium $[4,7]$.

$\varepsilon_{H S}^{L}=\varepsilon_{1}\left(\left(1+v_{2}\right) \varepsilon_{2}+\left(1-v_{2}\right) \varepsilon_{1}\right) /\left(\left(1-v_{2}\right) \varepsilon_{2}+\left(1+v_{2}\right) \varepsilon_{1}\right) ;$

$\varepsilon_{H S}^{U}=\varepsilon_{2}\left(\left(1+v_{1}\right) \varepsilon_{1}+\left(1-v_{1}\right) \varepsilon_{2}\right) /\left(\left(1-v_{1}\right) \varepsilon_{1}+\left(1+v_{1}\right) \varepsilon_{2}\right)$.

Since the honeycomb cores with absorbing coatings consist of three phase media, the multi-step equivalence is executed. Firstly, the air and absorbing coatings are equivalent to medium 1 . The matrix is regarded as medium 2. And then media 1 and 2 are homogenized. Therefore, there are four combining cases according to different effective sequences of upper and lower bounds

\subsection{Strong fluctuation theory (SFT)}

The constitutive matrix of random medium in Maxwell equations is a function of position. The effective electromagnetic properties depend on the correlation function, which is used to describe the randomness of scattering characteristics. The value of correlation function approaches zero when the size of phase materials is far less than the wavelength. The honeycomb cores with absorbing coatings are distributed periodically and uniformly in the $x-y$ plane. The same orientation is along the unit cell axis. The size of phase materials is less than a quarter of wave length in the $\mathrm{X}$ wave band $(8-12 \mathrm{GHz})$. A given accuracy of field magnitude is achieved [25]. The effective permittivity $\varepsilon_{e}$ in the zero-order approximation is determined by the following equation:

$\sum_{i=1}^{n} v_{i}\left(\varepsilon_{i}-\varepsilon_{e}\right) /\left(\varepsilon_{i}+\varepsilon_{e}\right)=0$

where $\varepsilon_{i}$ is the permittivity of the $i$-th phase medium and $v_{i}$ is the corresponding volume fraction. As there are two phase materials $(n=2)$, the effective permittivity is calculated by

$\varepsilon_{e}=\frac{1}{2}\left[\left(v_{1}-v_{2}\right)\left(\varepsilon_{1}-\varepsilon_{2}\right)+\sqrt{\left(v_{1}-v_{2}\right)^{2}\left(\varepsilon_{1}-\varepsilon_{2}\right)^{2}+4 \varepsilon_{2}}\right]$.

According to the layered model, the effective permittivity of honeycomb cores with absorbing coatings can be obtained by two-step equivalence from Eq. 4 .

The effective results by SFT and H-S upper and lower bounds are shown in Fig. 2. In cases 1 and 2, the final effective permittivities are on the basis of the upper bound in the first equivalence. In cases 3 and 4, the final effective permittivities are on the basis of the lower bound in the first equivalence. When the thickness of absorbing coatings is taken as the specific value at the intersecting point, the upper bound equals to the lower one in the second equivalence. In order to guarantee that the effective values by SFT lie between the upper and lower bounds, the effective H-S upper and lower bounds should be respectively taken as the maximum values from cases 1 and 2 and the minimum values from cases 3 and 4 . It is also known that the effective boundary values of honeycomb cores mainly depend on the equivalent upper and lower bounds of air and absorbing coating. Moreover, it is noted that the difference of effective permittivities becomes gradually large with the increase of coating thickness. It indicates that the absorbing coatings play the principal role in the effective electromagnetic properties.

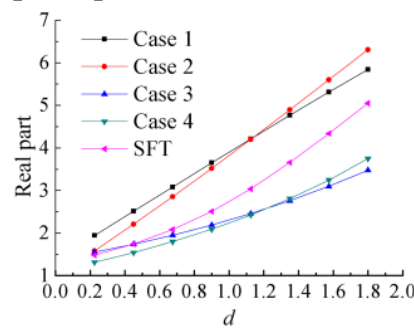

a

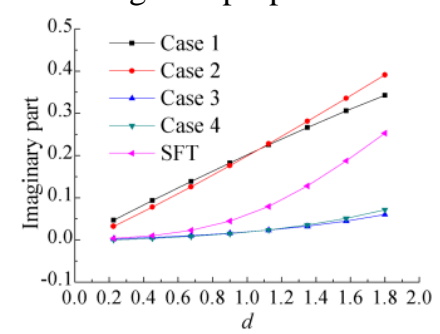

b
Fig. 2. Comparison of effective permittivities

\subsection{NRW method}

This method is established on the inversion of $S$ parameters from experimental measurement. Firstly, the reflection $(\Gamma)$ and transmission $(T)$ coefficients are calculated by Equations (5) and (7) after the reflection $\left(S_{11}\right)$ and transmission $\left(S_{21}\right)$ coefficients are measured. 
$\Gamma=K \pm \sqrt{K^{2}-1}$

$K=\left(S_{11}^{2}-S_{21}^{2}+1\right) /\left(2 S_{11}\right)$;

$T=\left(S_{11}+S_{21}-\Gamma\right) /\left(1-\left(S_{11}+S_{21}\right) \Gamma\right)$;

$\frac{1}{\Lambda^{2}}=\left[\frac{j}{2 \pi h} \ln \left(\frac{1}{T}\right)\right]^{2}$,

where $h$ is the thickness of measured sample as shown in Fig. $1 \mathrm{c}$.

The complex permittivity and permeability can be determined by $\Gamma$ and $T$ as given in Eq. 9 and Eq. 10 .

$$
\begin{aligned}
& \mu_{r}=(1+\Gamma) /\left(\Lambda(1-\Gamma) \sqrt{1 / \lambda_{0}^{2}-1 / \lambda_{c}^{2}}\right) ; \\
& \varepsilon_{r}=\lambda_{0}^{2}\left(1 / \Lambda^{2}+1 / \lambda_{c}^{2}\right) / \mu_{r},
\end{aligned}
$$

where $\lambda_{0}$ is the free-space wavelength and $\lambda_{c}$ is the cut-off wavelength.

The experimental honeycomb cores with absorbing coatings are shown in Fig. 3.

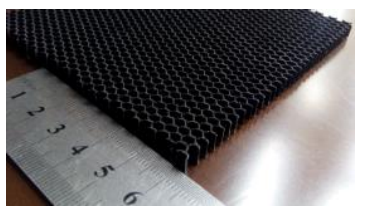

Fig. 3. Honeycomb core materials with absorbing coatings

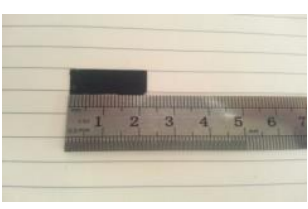

a

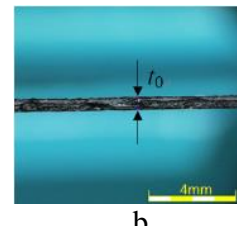

b
Fig. 4. Absorbing material: $a$ - tested sample; $b$ - wall thickness

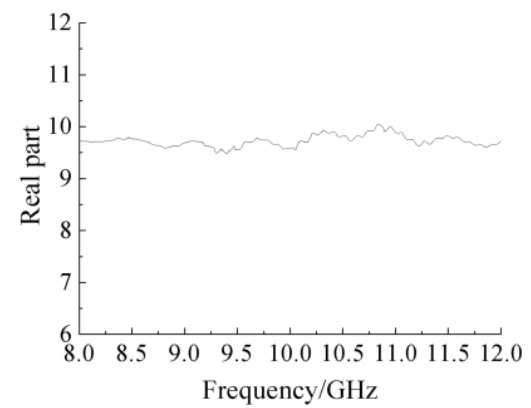

Fig. 5. Effective permittivity of absorbing material

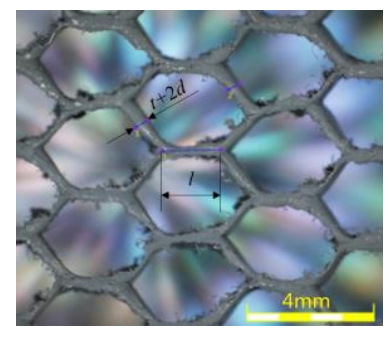

a

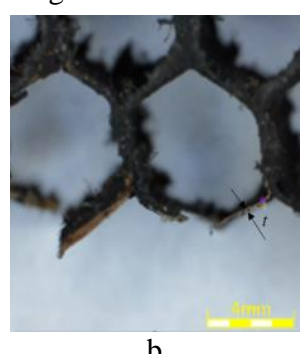

b
Fig. 6. Tested sample of honeycomb cores with absorbing coatings

The matrix is aramid paper which permittivity $\varepsilon_{m}$ equals to 1.23 . The geometries are measured by Olympus DSX100 Opto-Digital Microscope and the scattering parameters are measured by AV3629D Microwave Vector Network Analyzer. First of all, the permittivity of absorbing coatings is calculated after measuring scattering parameters. A piece of pure absorbing materials is taken for test as shown in Fig. 4. The thickness is $t_{0}=0.677 \mathrm{~mm}$. The effective permittivity is shown in Fig. 5. The mean permittivity (real part) is taken as $\varepsilon_{c}=9.78$. The honeycomb cores with absorbing coatings are shown in Fig. 6, the side length is $l=1.83 \mathrm{~mm}$; the thickness of matrix is $t=0.067 \mathrm{~mm}$; the thickness of absorbing coatings is $d=0.197 \mathrm{~mm}$; the axial depth is $h=5 \mathrm{~mm}$. As showed in Fig. 7, the red line represents the effective permittivity.

\subsection{Numerical simulation}

The simulation model, which is built in the CST Microwave Studio environment, is shown in Fig. 1 c. The model sizes are same with the experiment sample. The computational formulas of effective electromagnetic parameters are obtained from the analytic expression of $\mathrm{S}$ matrix elements which are found from the elements of the transfer matrix [22].

$$
\begin{aligned}
& S_{21}=S_{12}=1 /(\cos (n k h)-i(z+1 / z) \sin (n k h) / 2) ; \\
& S_{11}=S_{22}=i(1 / z-z) \sin (n k h) S_{21} / 2,
\end{aligned}
$$

where $n$ is the refractive index and $z$ is the wave impedance of homogenous medium.

Eq. 11 and Eq. 12 can be inverted to find $n$ and $z$ in terms of the scattering parameters as follows:

$$
\begin{aligned}
& n=\cos ^{-1}\left[\left(1-S_{11}^{2}+S_{21}^{2}\right) /\left(2 S_{21}\right)\right] /(k h) ; \\
& z=\sqrt{\left(\left(1+S_{11}\right)^{2}-S_{21}^{2}\right) /\left(\left(1-S_{11}\right)^{2}-S_{21}^{2}\right)} .
\end{aligned}
$$

According to the relation between $n$ and $z$, the effective permittivity and permeability of homogenous medium are as follows:

$\varepsilon_{e}=n / z, \mu_{e}=n z$.

The effective permittivity of honeycomb cores with absorbing coatings is $\varepsilon_{e}=1.61$ from the simulation results.

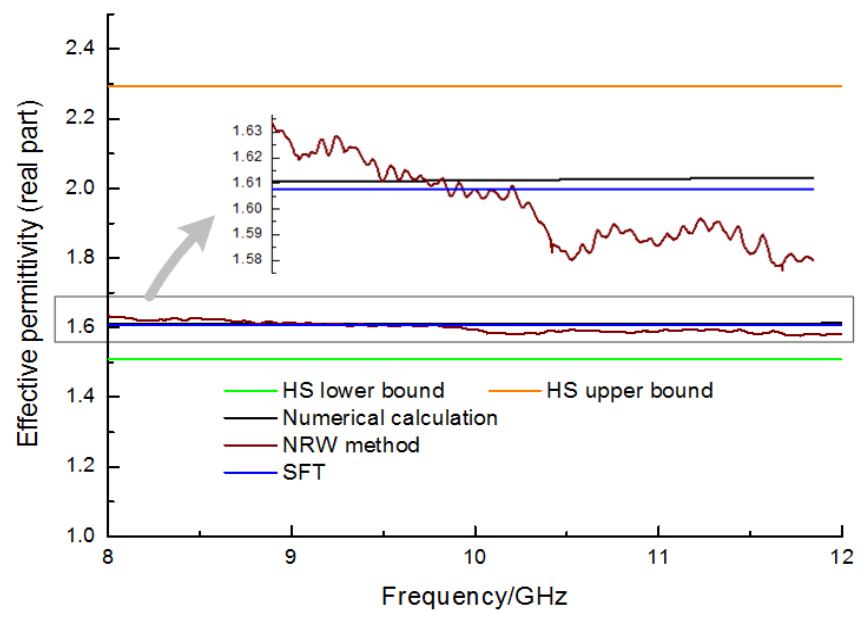

Fig. 7. Comparison of different methods

With the same model, the effective permittivity is 1.6 by SFT. And the H-S upper and lower bounds are also regained. From Fig. 7, the effective results have a good correspondence. In NRW method, the effective permittivities are fluctuated slightly because of the measured $S$ parameters. And all these effective permittivities lie between the H-S upper and lower bounds. 


\subsection{Improved strong fluctuation theory}

Here, the model characterization in section 2.1 is used again. The absorbing coatings and air are equivalent to homogenous dielectric by Eq. 4 . The effective permittivity $\varepsilon_{r}$ equals to 5.33. When the matrix of honeycomb cores with absorbing coatings is metallic materials, the wave propagation inside each unit cell is affected by the metallic walls. They can be equivalent to metallic waveguides. The propagation constants are dependent on the size parameters of waveguide structures.

The cut-off frequency of the waveguide with the arbitrary cross-section geometry is calculated from the surface variation $\Delta S$ by using the perturbation technique.

$f_{o h}^{2}=f_{o}^{2}(1-\Delta S / S)$.

As shown in Fig. 8, the hexagonal cell is perturbed into the rectangle or circumscribed circle waveguide structures.

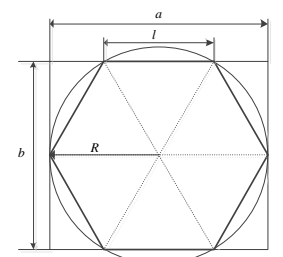

Fig. 8. Hexagonal cell and its perturbations

The effective permittivity of the rectangular waveguide structure filled with homogenous dielectric is expressed as [6]:

$\varepsilon_{\text {effw } 1}=\varepsilon_{r}\left(1-\omega_{0}^{2} / \omega^{2}\right)-j \sigma / \omega \varepsilon_{0}$.

The cut-off frequency of the rectangular waveguide is

$f_{o r}=\omega_{0} / 2 \pi=c_{0} \sqrt{(m / 2 a)^{2}+(n / 2 b)^{2}} / \sqrt{\varepsilon_{r}}$.

When Eq. 17 is used for the hexagonal cell, the effective permittivity for the dominant mode is expressed as

$\varepsilon_{\text {effh } 1}=\varepsilon_{r}-5(\pi / a)^{2}\left(c_{0} / \omega\right)^{2} / 4$.

According to Eq. 16, the cut-off frequency of hexagonal cells for the dominant mode is

$f_{\text {oh } 1}=\sqrt{5 / 4} f_{\text {or }}=6.289 \mathrm{GHz}$.

In Eq. 18 and Eq. 19, $a=l(1+2 \cos \alpha), \alpha=\pi / 3$.

The effective permittivity of the circular waveguide structure filled with the homogenous dielectric is expressed as

$\varepsilon_{\text {effw } 2}=\varepsilon_{r}-\left(p_{m n}^{\prime} / R\right)^{2} c_{0}^{2} / \omega^{2}-j \sigma / \omega \varepsilon_{0}$.

The cut-off frequency of the circular waveguide is

$f_{o c}=c_{0} p_{n m}^{\prime} /\left(2 \pi R \sqrt{\varepsilon_{r}}\right)$.

When the hexagonal cell is perturbed into the circumscribed circular waveguide structure, the effective permittivity for the dominant mode is expressed as

$\varepsilon_{\text {effh } 2}=\varepsilon_{r}-1.173\left(p_{n m}^{\prime} / R\right)^{2}\left(c_{0} / \omega\right)^{2}-j \sigma / \omega \varepsilon_{0}$.

The cut-off frequency of the circular waveguide is

$f_{o h 2}=\sqrt{1.173} f_{o c}=14.84 \mathrm{GHz}$,

where $R=1$.
For the dominant mode, $p_{n m}^{\prime}=p_{01}^{\prime}=3.832$ [26].

The depth of absorbing coatings is taken as $d=1.8 \mathrm{~mm}$. The effective results are shown in Fig. 9 from 5 to $30 \mathrm{GHz}$. The effective values of honeycomb cores with absorbing coatings approach that of the filled homogeneous dielectric. It indicates that the effective permittivity of honeycomb cores is much smaller than that of absorbing materials. The dispersion effect of effective permittivity attenuates with the increase of frequency.

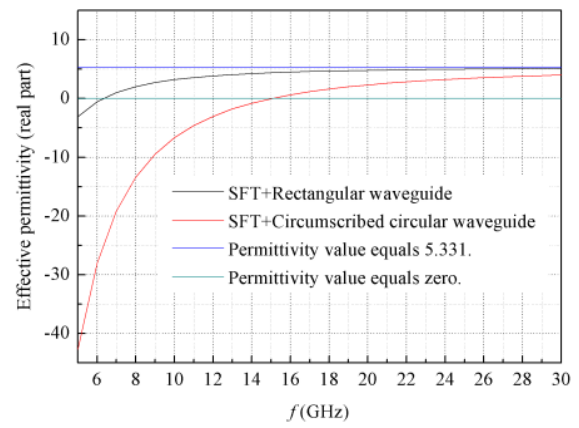

Fig. 9. Comparison of effective permittivities

\subsection{Discussion}

As a type of multi-phase materials, honeycomb cores with absorbing coatings can be equivalent step by step to uniform dielectric materials. They can also be regarded as the special random media which are homogenized by SFT. With comparison to the transmission/reflection method based on experimental measurement or numerical simulation, the effective results have a good agreement. It is proved that the effective calculations are valid and practically significant. When the matrix of honeycomb cores is equivalent to the rectangular and circumscribed circular metallic waveguide structures by the improved SFT, the dispersion effect of effective permittivity appears clearly. The effective results also show that the size and shape parameters of waveguide structures affect propagation properties directly.

\section{OPTIMIZATION PROBLEM}

\subsection{Description of absorption properties}

Generally, absorption properties are characterized by reflectivity or absorbing index. They depend mainly on electromagnetic properties of absorbing materials and geometries of absorbing structures.

The reflectivity is expressed as

$$
\begin{aligned}
& R=20 \lg \left|\left(z-z_{0}\right) /\left(z+z_{0}\right)\right| ; \\
& z=\sqrt{\left(\left(1+S_{11}\right)^{2}-S_{21}^{2}\right) /\left(\left(1-S_{11}\right)^{2}-S_{21}^{2}\right)},
\end{aligned}
$$

where $z$ is the wave impedance of effective medium and $z_{0}$ is the air impedance.

The absorbing index is expressed as

$$
A=P_{a b s} / P_{i n}=1-\left|S_{11}\right|^{2}-\left|S_{21}\right|^{2} \text {, }
$$

where $P_{a b s}$ is the absorbed power and $P_{i n}$ is the incident power.

In order to improve the impedance matching and efficiently optimize absorption properties, the reflection index is chosen as the optimization objective. 


\subsection{Optimization formulation}

Through analyzing the influences of thickness $d$ of absorbing coatings, axial length $h$ of honeycomb cores and side length $l$ on the reflection index respectively, it is found that the best parameter combination can generate the optimal absorbing properties. In order to seek the optimal scheme, the genetic algorithms (GAs) based on the global search are used.

The $90 \%$ absorption lies in the X-band under $-10 \mathrm{~dB}$ reflection loss [27]. When the reflection index $R$ is less than $-10 \mathrm{~dB}$, the absorption property mainly depends on the bandwidth. As shown in Fig. 10, when the area $T$ becomes greater, which is surrounded by the effective reflectivity curve and the boundary line $\left(R_{0}=-10 \mathrm{~dB}\right)$, the absorption property is better between the bandwidth of $\omega_{1}$ and $\omega_{2}$.

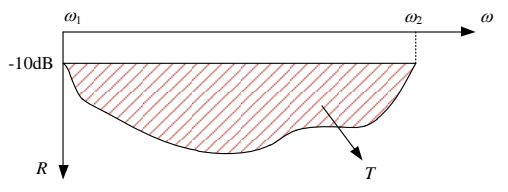

Fig. 10. Description of objective function

First of all, it is assured that the reflectivity $R$ of optimized structures is less than $-10 \mathrm{~dB}$ within the given bandwidth. The optimization objective is to maximize the area $T$ under the constraints of sizes and volume fraction. The optimization problem can be formulated as follows: Maximize $: T(l, h, d)$

Subject to $: R(\omega) \leq R_{0}$

$$
\begin{aligned}
& \text { vf } \leq 30 \% \\
& 3.0 \leq h \leq 8.0,1.0 \leq l \leq 3.0,0.1 \leq d \leq 0.3
\end{aligned}
$$

The objective function is

$T=\int_{\omega_{1}}^{\omega_{2}} \rho(\omega)\left|R(\omega)+R_{0}\right| d \omega$,

where $\rho(\omega)$ is the weight function.

Assuming that

$$
f(\omega)=\rho(\omega)\left|R(\omega)+R_{0}\right| \text {. }
$$

According to the compound quadrature formula, the objective function is approximated as

$$
T=\frac{\omega_{1}-\omega_{2}}{2 n}\left[f\left(\omega_{1}\right)+2 \sum_{i=1}^{n-1} f\left(\omega_{i}\right)+f\left(\omega_{2}\right)\right],
$$

where

$$
\omega_{i}=\omega_{1}+i\left(\omega_{2}-\omega_{1}\right) / n \quad(i=0,1,2 \cdots n) .
$$

The volume fraction of the coating is

$V f_{c}=\frac{[l(1+\cos \theta)+(2 / \sin \theta-1 / \tan \theta)(l \sin \theta-t-d)] d}{l^{2}(1+\cos \theta) \sin \theta}$

The volume fraction of the matrix is

$V f_{m}=\frac{(l(1+\cos \theta)-t / \sin \theta+t /(2 \tan \theta))(l \sin \theta-t / 2)}{l^{2}(1+\cos \theta) \sin \theta}$

The total volume fraction of solid materials is $V f=V f_{c}+V f_{m}$.

\subsection{Optimization results}

Since the relation between objective function and design variables cannot be expressed explicitly, the optimized designs are iteratively generated using genetic algorithms (GAs) which are motivated by the principles of natural genetics and selection [28].

Table 1. Design variables

\begin{tabular}{|c|c|c|c|c|}
\hline $\begin{array}{c}\text { Design } \\
\text { variables }\end{array}$ & $\begin{array}{c}\text { Lower } \\
\text { boundaries }\end{array}$ & $\begin{array}{c}\text { Initial } \\
\text { values }\end{array}$ & $\begin{array}{c}\text { Convergence } \\
\text { values }\end{array}$ & $\begin{array}{c}\text { Upper } \\
\text { boundaries }\end{array}$ \\
\hline$h, \mathrm{~mm}$ & 3.0 & 5.0 & 6.20 & 8 \\
\hline$l, \mathrm{~mm}$ & 1.0 & 2.0 & 2.576 & 3.0 \\
\hline$d, \mathrm{~mm}$ & 0.1 & 0.2 & 0.103 & 0.3 \\
\hline
\end{tabular}

The final convergence values are listed in Table 1. Convergence processes are shown in Fig. 11.
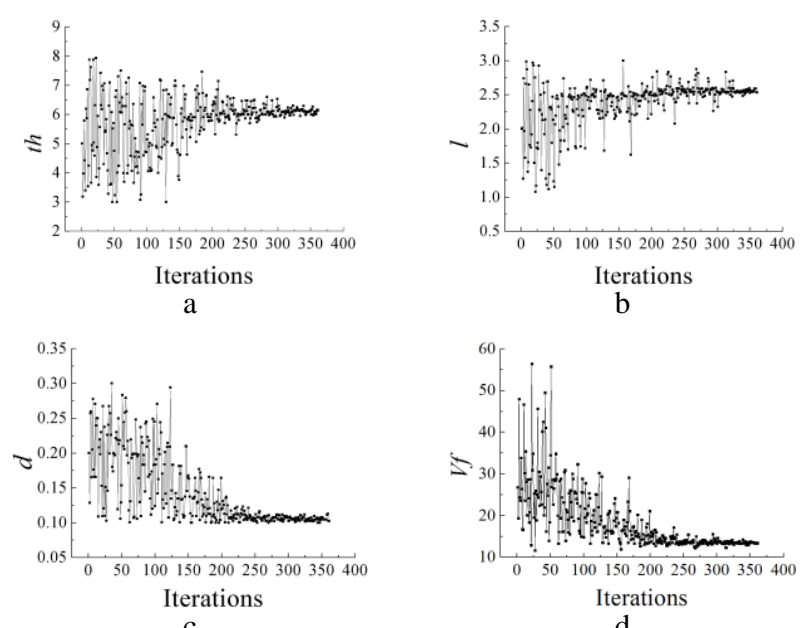

Fig. 11. Convergence processes of a-axial length $h$ of honeycomb core; $\mathrm{b}$-side length $l$; $\mathrm{c}$-thickness $d$ of absorption coat; $\mathrm{d}$ - total volume fraction $V f$

The minimum reflection index $R$ equals -35.41 at the frequency point $f=10.25 \mathrm{GHz}$ when the iteration converges. The optimal effective reflectivity curve within the X-band is shown in Fig. 12.

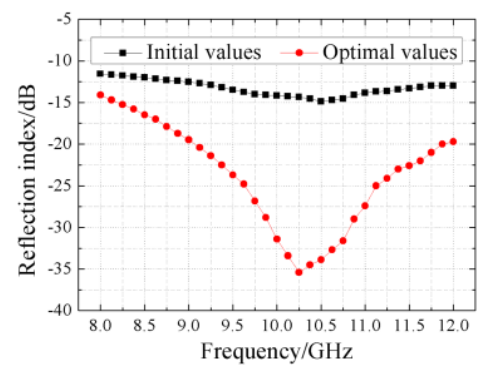

Fig. 12. Optimal absorbing properties

\section{CONCLUSIONS}

In this paper, we firstly predicted the effective permittivity of honeycomb cores with absorbing coatings by experimental measurement, numerical simulation and theoretical calculation. The effective results show a good agreement. The upper and lower bounds were calculated by the multi-step equivalence when the honeycomb cores with absorbing coatings are regarded as the multiphase materials. And the effective values obtained directly by the strong fluctuation theory lie between the H-S upper and lower bounds. It indicates that these methods are feasible for calculations of the effective electromagnetic parameters of honeycomb cores with absorbing coatings. Furthermore, the cut-off frequency besides the effective permittivity was obtained by using the improved strong fluctuation theory. 
In the meantime, the dispersion effect was observed. Lastly the absorption properties were optimized under the upper bound constraints of volume and reflectivity within the given frequency range. The minimum reflection index was gained in the special frequency point.

\section{Acknowledgments}

This work is supported by National Natural Science Foundation of China (11372250, 11002112), Aeronautical Science Foundation of China (2012ZA53011) and Fundamental Research Funds for the Central Universities (3102014JCS05006).

\section{REFERENCES}

1. Fan, H.L., Wang, W., Chao, Z.M. Microwave Absorbing Eomposite Lattice Grids Composites Science and Technology 67 2007: pp. 3472-3479.

http://dx.doi.org/10.1016/j.compscitech.2007.03.002

2. Kopyt, P., Damian, R., Celuch, M., Ciobanu, R. Dielectric Properties of Chiral Honeycombs-Modelling and Experiment Composites Science and Technology 70 2010: pp. $1080-1088$.

3. Smith, F.C., Scarpa, F. Design of Honeycomb-like Composites for Electromagnetic and Structural Applications IEE Proceedings-Microwaves Antennas and Propagation 151 (1) 2004: pp. $9-15$.

http://dx.doi.org/10.1109/TAP.2004.841320

4. Johansson, M., Holloway, C.L., Kuester, E.F. Effective Electromagnetic Properties of Honeycomb Composites, and Hollow-pyramidal and Alternating-wedge Absorbers IEEE Transactions on Antennas and Propagation 53 (2) 2005: pp. $728-736$.

5. Smith, F.C. Effective permittivity of dielectric honeycombs IEE Proceedings-Microwaves Antennas and Propagation 146 (1) 1999: pp. 55-59.

http://dx.doi.org/10.1049/ip-map:19990392

6. Smith, F.C., Scarpa, F., Chambers, B. The Electromagnetic Properties of Re-Entrant Dielectric Honeycombs IEEE Microwave and Guided Wave Letters $10(11)$ 2000: pp. $451-453$. http://dx.doi.org/10.1109/75.888829

7. Kuester, E.F., Holloway, C. Comparison of Approximations for Effective Parameters of Artificial Dielectrics IEEE Transactions on Microwave Theory and Techniques 38 (11) 1990: pp. $1752-1755$. http://dx.doi.org/10.1109/22.60028

8. Spiller, E. Low-loss Reflection Coatings Using Absorbing Materials Applied Physics Letters 20 (9) 1972: pp. $365-367$. http://dx.doi.org/10.1063/1.1654189

9. Cao, M.S., Qin, R.R., Qiu, C.J., Zhua, J. Matching Design and Mismatching Analysis towards Radar Absorbing Coatings Based on Conducting Plate Materials and Design 24 2003: pp. 391-396.

10. Liu, L.D., Duan, Y.P., Ma, L.X., Liu, S.H., Yu, Z. Microwave Absorption Properties of a Wave-absorbing Coating Employing Carbonyl-iron Powder and Carbon Black Applied Surface Science 257 2010: pp. 842-846.

11. He, Y.F., Gong, R.Z., Cao, H., Wang, X., Zheng, Y. Preparation and Microwave Absorption Properties of Metal Magnetic Micropowder-coated Honeycomb Sandwich Structures Smart Materials and Structures 16 2007: pp. $1501-1505$.

12. Zhou, P.H., Huang, L.R., Xie, J.L., Liang, D.F., Lu, H.P., Deng, L.J. A Study on the Effective Permittivity of Carbon/PI
Honeycomb Composites for Radar Absorbing Design IEEE Transactions on Antennas and Propagation 60 (8) 2012: pp. $3679-3683$.

http://dx.doi.org/10.1109/TAP.2012.2201120

13. Kuester, E.F., Holloway, C.L. A Low-frequency Model for Wedge or Pyramid Absorber Arrays-I: Theory IEEE Transactions on Electromagnetic Compatibility $36(4)$ 1994: pp. 300-306. http://dx.doi.org/10.1109/15.328859

14. Smith, D.R., Pendry, J.B. Homogenization of Metamaterials by Field Averaging Journal of the Optical Society of America B 23 (3) 2006: pp. 391-403. http://dx.doi.org/10.1364/JOSAB.23.000391

15. Amirkhizi, A.V., Nemat-Nasser, S. Microstructurally-based Homogenization of Electromagnetic Properties of Periodic Media C. R. Mecanique 336 2008: pp. 24-33. http://dx.doi.org/10.1016/j.crme.2007.10.012

16. Wang, H., Pulliainen, J., Hallikainen, M. Application of Strong Fluctuation Theory to Microwave Emission from Dry Snow Progress in Electromagnetics Research 29 2000: pp. 39-55.

17. Lakhtakia, A. Application of Strong Permittivity Fluctuation Theory for Isotropic, Cubically Nonlinear, Composite Mediums Optics Communications 192 (3) 2001: pp. 145-151. http://dx.doi.org/10.1016/S0030-4018(01)01202-0

18. Peng, Z.H., Cao, M.S., Yuan, J., Xiao, G. Strong Fluctuation Theory for Effective Electromagnetic Parameters of Fiber Fabric Radar Absorbing Materials Materials and Design 25 (5) 2004: pp. 379-384.

19. Naseri, F., Hossein, S. Investigation of Linear and Non-linear Properties of Oxide Glass-metal Nanoparticle Composites by the Strong-permittivity-fluctuation theory Optics Communications 309 2013: pp. 134-138. http://dx.doi.org/10.1016/j.optcom.2013.06.005

20. Nicolson, A.M., Ross, G.F. Measurement of the Intrinsic Properties of Materials by Time-domain Techniques IEEE Transactions on Instrumentation and Measurement 19 (4) 1970: pp. $377-382$.

21. Weir, W.B. Automatic Measurement of Complex Dielectric Constant and Permeability at Microwave Frequencies Proceedings of the IEEE 62 (1) 1974: pp. 33-36.

22. Smith, D.R., Vier, D.C., Koschny, T.C., Soukoulis, M. Electromagnetic Parameter Retrieval from Inhomogeneous Metamaterials Physical Review E 71(3) 2005: pp. 1-11.

23. Hashin, Z., Shtrikman, S. A Variational Approach to the Theory of the Effective Magnetic Permeability of Multiphase Materials Journal of Applied Physics 33 1962: pp. 3125 - 3131 .

24. Tsang, L., Kong, J.A. Scattering of Electromagnetic Waves from Random Media with Strong Permittivity Fluctuations Radio Science 16 (3) 1981: pp. 303-320.

25. Sarabandi, K., Siqueira, P.R. Numerical Scattering Analysis for Two-dimensional Dense Random Media: Characterization of Effective Permittivity IEEE Transactions on Antennas and Propagation 45 (5) 1997: pp. $858-867$. http://dx.doi.org/10.1109/8.575638

26. David, M. Pozar. Microwave Engineering (Fourth Edition), JohnWiley \& Sons, 2012.

27. Lee, W.J., Lee, J.W., Kim, C.G. Characteristics of an Electromagnetic Wave Absorbing Composite Structure with a Conducting Polymer Electromagnetic Bandgap (EBG) in the Xband Composites Science and Technology 68 (12) 2008: pp. $2485-2489$.

28. Holland, J. Adaptation in Natural and Artificial Systems, University of Michigan Press, 1975. 